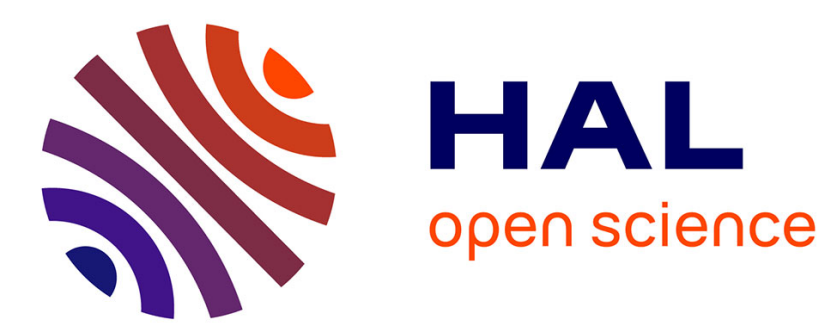

\title{
A direct Poisson solver in spherical geometry with an application to diffusiophoretic problems
}

Te-Sheng Lin, Wei-Fan Hu, Chaouqi Misbah

\section{To cite this version:}

Te-Sheng Lin, Wei-Fan Hu, Chaouqi Misbah. A direct Poisson solver in spherical geometry with an application to diffusiophoretic problems. Journal of Computational Physics, 2020, 409, pp.109362. 10.1016/j.jcp.2020.109362 . hal-03083603

\section{HAL Id: hal-03083603 https://hal.science/hal-03083603}

Submitted on 19 Dec 2020

HAL is a multi-disciplinary open access archive for the deposit and dissemination of scientific research documents, whether they are published or not. The documents may come from teaching and research institutions in France or abroad, or from public or private research centers.
L'archive ouverte pluridisciplinaire HAL, est destinée au dépôt et à la diffusion de documents scientifiques de niveau recherche, publiés ou non, émanant des établissements d'enseignement et de recherche français ou étrangers, des laboratoires publics ou privés. 


\title{
A direct Poisson solver in spherical geometry with an application to diffusiophoretic problems
}

\author{
Te-Sheng Lin $^{\mathrm{a}}$, Wei-Fan $\mathrm{Hu}^{\mathrm{b}}$, Chaouqi Misbah ${ }^{\mathrm{c}}$ \\ ${ }^{a}$ Department of Applied Mathematics, National Chiao Tung University, Hsinchu 30010, Taiwan \\ ${ }^{b}$ Department of Applied Mathematics, National Chung Hsing University, Taichung 40227, Taiwan \\ ${ }^{c}$ Université Grenoble Alpes, CNRS, LIPhy, F-38000 Grenoble, France
}

\begin{abstract}
We propose a simple and efficient class of direct solvers for Poisson equation in finite or infinite domains related to spherical geometry. The solver was developed based on truncated spherical harmonics expansion, where the differential mode equations were solved by second-order finite difference method without handling coordinate singularities. The solver was further extended to study the dynamics of a diffusiophoretic particle suspended in Stokes flow. Numerical experiments suggested that the particle can achieve a self-sustained unidirectional motion at moderate Péclet numbers, whereas the particle motion becomes chaotic in high Péclet number regimes. The statistical analysis illustrates the run-and-tumble-like nature at short times and diffusive nature at long times without any source of noise.
\end{abstract}

Keywords: Fast Poisson solver, spherical harmonics expansion, diffusiophoresis, microswimmer 2010 MSC: $65 \mathrm{~N} 06,65 \mathrm{~N} 35,76 \mathrm{D} 07$

\section{Introduction}

The design of artificial microswimmers has become an active research area in the past decade. A prototypical example, Janus particle, named after the two-faced Roman god, uses the asymmetry property of the active surface to generate motions [1]. It was shown by Michelin et al. 2] that, under the framework of axisymmetric assumption, the anisotropic design is not necessary for locomotion, whereas a spontaneous autophoretic directed motion can be activated for an isotropic particle when the Péclet number is above a critical number. In this Note, we numerically examine the dynamics of the diffusiophoretic system in the full three-dimensional model. Interestingly, we observe a transition to chaotic dynamics by increasing the Péclet number further. Such results show evidence that the complicated motions may already be hidden in purely isotropic media due to the intrinsic nonlinearities of the problem.

To investigate the dynamics of diffusiophoretic particles, we first develop a direct numerical solver for Poisson equation in a unit sphere based on spherical harmonics finite difference method. It is known that, in spherical coordinates, appropriate conditions need to be imposed at the coordinate singularities to have the desired accuracy (see, for example, the discussion by Shen [3). We avoid placing grid points directly on the coordinate singularities by arranging properly the grid points and thus the numerical scheme does not require any pole conditions. A similar trick based on the combination of Fourier series expansions and finite difference approaches was proposed by Lai et al. [4]. We then solve Poisson equation in an infinite domain by using Kelvin's inversion which maps the domain into a finite one with the perfect preservation of the Laplace operator. As a result, the proposed fast solver can be straightforwardly applied.

This Note is organized as follows: The description of the solver is presented in Section 2 and in Section 3 we show our findings on the dynamics of a diffusiophoretic particle. Some concluding remarks and future works are given in Section 4 .

Email addresses: tslin@math.nctu.edu.tw (Te-Sheng Lin), wfhu@nchu.edu.tw (Wei-Fan Hu), chaouqi.misbah@univ-grenoble-alpes.fr (Chaouqi Misbah) 


\section{Fast Poisson solver in spherical geometry}

Under the spherical coordinate system (radial distance $r$, polar angle $\theta$, and azimuthal angle $\phi$ ), the Poisson equation is written as

$$
\Delta c(r, \theta, \phi)=\frac{1}{r^{2}} \frac{\partial}{\partial r}\left(r^{2} \frac{\partial c}{\partial r}\right)+\frac{1}{r^{2} \sin \theta} \frac{\partial}{\partial \theta}\left(\sin \theta \frac{\partial c}{\partial \theta}\right)+\frac{1}{r^{2} \sin ^{2} \theta} \frac{\partial^{2} c}{\partial \phi^{2}}=f(r, \theta, \phi),
$$

where $c$ is an unknown function and $f$ is a given smooth function in which $\theta \in[0, \pi], \phi \in[0,2 \pi)$. Two types of domains are considered in this Note, namely, the finite spherical domain $(r \in[0,1])$ and the infinite domain $(r \in[1, \infty))$. For both cases, the boundary condition should be imposed on the spherical surface $(r=1)$; three different boundary conditions are considered, that is, Dirichlet $\left(c(1, \theta, \phi)=c^{D}(\theta, \phi)\right)$, Neumann $\left(\frac{\partial c}{\partial r}(1, \theta, \phi)=c^{N}(\theta, \phi)\right)$, and Robin type $\left(\frac{\partial c}{\partial r}(1, \theta, \phi)+\alpha c(1, \theta, \phi)=c^{R}(\theta, \phi)\right)$, where $c^{D}, c^{N}$ and $c^{R}$ are given functions and $\alpha$ is a constant. Notice that for the infinite domain case, we impose a vanishing condition $c(r \rightarrow \infty)=0$ to ensure the uniqueness of the solution. It is important to mention that our numerical scheme can treat all types of boundary conditions equally well as we can see from the implementation later.

\subsection{Finite domain $r \in[0,1]$}

Let us first consider the finite domain case. Due to the spherical coordinate representation (1), the singularities occur not only at the origin $(r=0)$, but also at the north $(\theta=0)$ and the south poles $(\theta=\pi)$. Therefore, how to handle those singularities properly becomes an important issue in numerical discretizations. Since the considered domain is related to spherical geometry, it is convenient to represent the solution by spherical harmonics expansion

$$
c(r, \theta, \phi)=\sum_{n=0}^{p} \sum_{m=0}^{n} P_{n}^{m}(\cos \theta)\left(c_{m n}(r) \cos m \phi+\tilde{c}_{m n}(r) \sin m \phi\right),
$$

where $P_{n}^{m}$ denotes the associated Legendre polynomial of order $m$ and degree $n[5$. Notice that the solution is truncated up to the spherical harmonics of order $p$ for numerical purpose. The coefficients in the spherical harmonics expansion (2) are given by

$$
\begin{aligned}
& c_{m n}(r)=\frac{(2 n+1)(n-m) !}{2(n+m) ! N(m)} \int_{0}^{2 \pi} \int_{0}^{\pi} c(r, \theta, \phi) P_{n}^{m}(\cos \theta) \cos m \phi \sin \theta \mathrm{d} \theta \mathrm{d} \phi, \\
& \tilde{c}_{m n}(r)=\frac{(2 n+1)(n-m) !}{2(n+m) ! N(m)} \int_{0}^{2 \pi} \int_{0}^{\pi} c(r, \theta, \phi) P_{n}^{m}(\cos \theta) \sin m \phi \sin \theta \mathrm{d} \theta \mathrm{d} \phi,
\end{aligned}
$$

where $N(0)=2 \pi$, and $N(m)=\pi$ for $m \neq 0$. One can verify that the spherical harmonic functions $Y_{n}^{m}(\theta, \phi) \equiv P_{n}^{m}(\cos \theta) e^{i m \phi}$ are the eigenfunctions of the Laplace-Beltrami operator with

$$
\Delta_{s} Y_{n}^{m}=-n(n+1) Y_{n}^{m}, \quad \text { where } \quad \Delta_{s}=\frac{1}{\sin \theta} \frac{\partial}{\partial \theta}\left(\sin \theta \frac{\partial}{\partial \theta}\right)+\frac{1}{\sin ^{2} \theta} \frac{\partial^{2}}{\partial \phi^{2}} .
$$

Substituting the spherical harmonics expansion (2) into the Poisson equation (1) and using Eq. (4), consequently, one can derive a set of one-dimensional ordinary differential equations

$$
\frac{1}{r^{2}} \frac{\mathrm{d}}{\mathrm{d} r}\left(r^{2} \frac{\mathrm{d} c_{m n}}{\mathrm{~d} r}\right)-\frac{n(n+1)}{r^{2}} c_{m n}=f_{m n} \quad \text { and } \quad \frac{1}{r^{2}} \frac{\mathrm{d}}{\mathrm{d} r}\left(r^{2} \frac{\mathrm{d} \tilde{c}_{m n}}{\mathrm{~d} r}\right)-\frac{n(n+1)}{r^{2}} \tilde{c}_{m n}=\tilde{f}_{m n},
$$

where $f_{m n}(r)$ and $\tilde{f}_{m n}(r)$ are obtained in a similar fashion to Eq. (3). Accordingly, the associated boundary conditions take the form: Dirichlet $c_{m n}(1)=c_{m n}^{D}$ and $\tilde{c}_{m n}(1)=\tilde{c}_{m n}^{D}$; Neumann $c_{m n}^{\prime}(1)=c_{m n}^{N}$ and $\tilde{c}_{m n}^{\prime}(1)=$ $\tilde{c}_{m n}^{N}$; or Robin type $c_{m n}^{\prime}(1)+\alpha c_{m n}(1)=c_{m n}^{R}$ and $\tilde{c}_{m n}^{\prime}(1)+\alpha \tilde{c}_{m n}(1)=\tilde{c}_{m n}^{R}$. The above boundary values can also be found by using Eq. (3). 
The discretized variables are defined on $(p+1)$ nodes along the $\theta$-direction and $2(p+1)$ uniformly distributed nodes $\phi_{k}=k \frac{2 \pi}{2(p+1)}$ in the $\phi$-direction, so that $\theta_{j}=\cos ^{-1}\left(s_{j}\right)$, where $s_{j}$ 's are $(p+1)$-point Gauss-Legendre quadrature nodes. Under such a $\theta-\phi$ grid layout, the numerical quadrature for the integrations in Eq. (3) converges superalgebraically via the combination of Gaussian quadrature (in $\theta$-direction) and trapezoidal rule (in $\phi$-direction). This step can be performed efficiently using the public software SPHEREPACK 6]. There are several advantages of using spherical harmonics expansion: (i) The number of Gauss-Legendre nodes can be taken small since the truncated series [2] is spectrally convergent [7]. (ii) The usage of Gauss-Legendre nodes naturally avoids placing the points directly at the poles $(\theta=0, \pi)$ where the singularities of the Poisson equation (1) taken place. (iii) The three-dimensional Poisson equation is converted to one-dimensional differential mode equations (5) that significantly reduces the numerical complexity of the problem. Furthermore, parallel computations on solving those mode equations can be implemented naturally. Now, the remaining issue is how to handle the coordinate singularity at $r=0$ for the mode equations (5).

To proceed, we choose a grid in the radial direction by setting the uniform grid half mesh away from the origin

$$
r_{i}=(i-1 / 2) \Delta r, \quad i=1,2, \cdots, M,
$$

with the mesh width $\Delta r=1 / M$. We deliberately avoid putting grid point at the origin directly. For succinctness, we will denote the discretized variables by $C_{i} \approx c_{m n}\left(r_{i}\right)$ and $F_{i} \approx f_{m n}\left(r_{i}\right)$. By multiplying the term $r^{2}$ on both sides of the differential mode equations in Eq. (5), and then apply second-order finite difference discretization, we obtain

$$
\left(\frac{r_{i-1 / 2}^{2}}{\Delta r^{2}}\right) C_{i-1}+\left(-\frac{r_{i-1 / 2}^{2}+r_{i+1 / 2}^{2}}{\Delta r^{2}}-n(n+1)\right) C_{i}+\left(\frac{r_{i+1 / 2}^{2}}{\Delta r^{2}}\right) C_{i+1}=r_{i}^{2} F_{i}, \quad i=1,2, \cdots M .
$$

The resulting matrix formed by the above discretizations has two advantages. One is being symmetric, which is consistent with the self-adjointness of the original differential operator. The other one is being tridiagonal so that the linear system can be solved easily via the Thomas algorithm.

When solving the linear system (7), the ghost values $C_{0}$ and $C_{M+1}$ remain to be specified. For $i=1$, one can immediately find that the coefficient for $C_{0}$ vanishes thanks to the setup of the radial grid (6), hence no extra condition at $r=0$ is required. The other ghost value $C_{M+1}$ can be found by the secondorder approximation for Dirichlet $\frac{C_{M+1}+C_{M}}{2}=c_{m n}^{D}$, Neumann $\frac{C_{M+1}-C_{M}}{\Delta r}=c_{m n}^{N}$, or Robin type boundary condition $\frac{C_{M+1}-C_{M}}{\Delta r}+\alpha \frac{C_{M+1}+C_{M}}{2}=c_{m n}^{R}$. A similar procedure can be straightforwardly applied for solving the coefficients $\tilde{c}_{m n}(r)$ and thus the repeated scheme is omitted here. We emphasize that since the finite difference discretization is adopted here, in the case where the solution has sharp transitions such as boundary layers or interior layers, to achieve the desired accuracy one can easily extend the present scheme to adaptive mesh with the same computational cost.

\subsection{Infinite domain $r \in[1, \infty)$}

Now we turn our attention to tackle the infinite domain problem. Here, we employ Kelvin's inversion 8 by introducing the radial coordinate transformation $\bar{r}=1 / r$ and further define the associated variables by $\bar{c}(\bar{r}, \theta, \phi)=\frac{1}{\bar{r}} c\left(\frac{1}{\bar{r}}, \theta, \phi\right)$ and $\bar{f}(\bar{r}, \theta, \phi)=\frac{1}{\bar{r}} f\left(\frac{1}{\bar{r}}, \theta, \phi\right)$ so that the considered domain will be finite $\bar{r} \in(0,1]$. After some careful calculations, the Poisson equation (1) becomes

$$
\bar{\Delta} \bar{c}(\bar{r}, \theta, \phi)=\frac{1}{\bar{r}^{2}} \frac{\partial}{\partial \bar{r}}\left(\bar{r}^{2} \frac{\partial \bar{c}}{\partial \bar{r}}\right)+\frac{1}{\bar{r}^{2} \sin \theta} \frac{\partial}{\partial \theta}\left(\sin \theta \frac{\partial \bar{c}}{\partial \theta}\right)+\frac{1}{\bar{r}^{2} \sin ^{2} \theta} \frac{\partial^{2} \bar{c}}{\partial \phi^{2}}=\frac{1}{\bar{r}^{4}} \bar{f}(\bar{r}, \theta, \phi) .
$$

Remarkably, we see that the Laplace operator is perfectly preserved through this coordinate transformation. Moreover, we should point out that the transformed solution $\bar{c}$ exists only when the solution admits the behavior $c(r \rightarrow \infty) \approx r^{-\beta}$ with $\beta \geq 1$. As a consequence $\bar{c}$ is bounded at $\bar{r}=0$ and it is not necessary to impose any additional condition here. Hence, to solve 8 we can just follow exactly the same procedure as presented in the previous subsection. 


\subsection{Numerical verification}

In this subsection we perform the convergence study of the proposed scheme. For the finite domain case $(r \in[0,1])$ we solve Poisson equation with exact solution $c_{e}(r, \theta, \phi)=\sin (10 r \sin \theta \cos \phi+\pi / 4) \sin (5 r \sin \theta \sin \phi+$ $\pi / 3) \sin (5 r \cos \theta+\pi / 2)$ and set the parameter $\alpha=1$ in the cases of Robin boundary condition (notice that the chosen solution is significantly oscillatory). For the infinite domain case $(r \in[1, \infty))$ we set $c_{e}(r, \theta, \phi)=r^{3} \cos \theta \sin ^{2} \theta \cos \phi \sin \phi / \exp \left(r^{2}\right)$ and $\alpha=-1$. We denote the numerical solutions by $c_{h}$ and show the results in Table 1. where the upper panel gives the mesh refinement results for the finite domain and the lower panel for the infinite domain. In each run we fix 32 Gauss-Legendre nodes for polar angle and 64 nodes for azimuthal angle. Notice that the discretization (7), with truncation error $O\left(\Delta r^{2}\right)$, introduces an error $\left\|c_{h}-c_{e}\right\|_{\infty}$ with order $O\left(c_{r r}(r=0) \Delta r^{2} \log \Delta r\right)+O\left(\Delta r^{2}\right)$. As expected, we obtain a roughly secondorder convergence for the finite domain case while a clean second-order of accuracy for the infinite domain case. In addition, these tests show the robustness of our proposed Poisson solver for dealing with oscillatory solutions. On the other hand, the computational cost for the present method is only $O\left(M p^{2}(\log p)^{2}\right)$; the CPU time for the finest mesh case $M=1024$ is just within one second on a MacBook Pro (2016), showing the efficiency of the present scheme.

\begin{tabular}{lcclllllc}
\hline \multirow{2}{*}{$M$} & \multicolumn{2}{c}{ Dirichlet } & & \multicolumn{2}{c}{ Neumann } & & \multicolumn{2}{c}{ Robin } \\
\cline { 2 - 3 } & $\left\|c_{h}-c_{e}\right\|_{\infty}$ & Rate & & $\left\|c_{h}-c_{e}\right\|_{\infty}$ & Rate & & $\left\|c_{h}-c_{e}\right\|_{\infty}$ & Rate \\
\hline 128 & $1.413 \mathrm{E}-03$ & - & & $1.624 \mathrm{E}-03$ & - & & $1.628 \mathrm{E}-03$ & - \\
256 & $4.271 \mathrm{E}-04$ & 1.73 & & $4.862 \mathrm{E}-04$ & 1.74 & & $4.810 \mathrm{E}-04$ & 1.76 \\
512 & $1.259 \mathrm{E}-04$ & 1.76 & & $1.416 \mathrm{E}-04$ & 1.78 & & $1.394 \mathrm{E}-04$ & 1.79 \\
1024 & $3.637 \mathrm{E}-05$ & 1.79 & & $4.046 \mathrm{E}-05$ & 1.81 & & $3.974 \mathrm{E}-05$ & 1.81 \\
\hline 128 & $1.465 \mathrm{E}-05$ & - & & $1.463 \mathrm{E}-05$ & - & & $1.464 \mathrm{E}-05$ & - \\
256 & $3.664 \mathrm{E}-06$ & 2.00 & & $3.660 \mathrm{E}-06$ & 2.00 & & $3.661 \mathrm{E}-06$ & 2.00 \\
512 & $9.158 \mathrm{E}-07$ & 2.00 & & $9.148 \mathrm{E}-07$ & 2.00 & & $9.150 \mathrm{E}-07$ & 2.00 \\
1024 & $2.290 \mathrm{E}-07$ & 2.00 & & $2.287 \mathrm{E}-07$ & 2.00 & & $2.288 \mathrm{E}-07$ & 2.00 \\
\hline
\end{tabular}

Table 1: Mesh refinement results with different types of boundary conditions for the finite domain (upper panel) and infinite domain cases (lower panel).

\section{Dynamics of a diffusiophoretic particle}

We consider the dynamics of a spherical shaped colloidal particle immersed in a Newtonian fluid. The fluid is assumed to be incompressible and obeys the Stokes equations. Surrounding solute is isotropically emitted from the particle surface. The solute interacts with the particle through a short-range potential with a characteristic length that is much less than the particle radius, leading to the so-called sharp interface limit amounting to a slip tangential velocity on the particle surface [9] (see below). The solute concentration is assumed to diffuse and be advected by the surrounding fluid flow.

We use the same scalings to non-dimensionalize the governing equations as in [2]. The dimensionless governing equations are described in the following: In the co-moving frame attached to the particle center, the fluid flow and solute concentration coupled system is modeled by

$$
\begin{array}{r}
-\nabla p+\Delta \mathbf{u}=\mathbf{0}, \quad \nabla \cdot \mathbf{u}=0 \\
\frac{\partial c}{\partial t}+\mathbf{u} \cdot \nabla c=\frac{1}{P e} \Delta c
\end{array}
$$

where $c$ stands for the solute concentration, the velocity field $\mathbf{u}=u_{r} \mathbf{e}_{r}+u_{\theta} \mathbf{e}_{\theta}+u_{\phi} \mathbf{e}_{\phi}\left(\mathbf{e}_{r}, \mathbf{e}_{\theta}\right.$ and $\mathbf{e}_{\phi}$ stand for the basis vectors in spherical coordinates, $u_{r}, u_{\theta}$ and $u_{\phi}$ are scalar functions), $p$ is the pressure and $P e$ is the Péclet number. The particle is suspended in an unbounded fluid domain $\Omega=\{(r, \theta, \phi) \mid r \in$ 
$[1, \infty), \theta \in[0, \pi], \phi \in[0,2 \pi)\}$. The tangential slip velocity on the particle surface $(r=1)$ is given by $\mathbf{u}(1, \theta, \phi)=\nabla_{s} c(1, \theta, \phi)$, where $\nabla_{s}$ denotes the surface gradient operator. The fixed-flux boundary condition of surface activity reads $\frac{\partial c}{\partial r}(1, \theta, \phi)=-1$, where the negative sign denotes the emission mechanism. In the far-field limit, the velocity field converges to the phoretic velocity $\mathbf{u}(r \rightarrow \infty)=-(\mathbf{U}+\boldsymbol{\Omega} \times \mathbf{r})$ and the concentration is assumed to attenuate by $c(r \rightarrow \infty)=0$. The phoretic kinematics of the particle can be arrived by using the reciprocal theorem [10], which allows the relation for the translational velocity $\mathbf{U}$ and rotational velocity $\Omega$ to the surface slip velocity via the surface integral on the unit sphere $S$ (in Cartesian components):

$$
\mathbf{U}=-\frac{1}{4 \pi} \int \mathbf{u}(r=1) \mathrm{d} S, \quad \boldsymbol{\Omega}=-\frac{3}{8 \pi} \int \mathbf{e}_{r} \times \mathbf{u}(r=1) \mathrm{d} S
$$

It can be deduced that the particle motion is always irrotational $(\boldsymbol{\Omega}=\mathbf{0})$ due to the imposed surface slip flow.

It is interesting to see that, a stationary solution where there is no net flow and zero phoretic velocity $\mathbf{U}=\mathbf{0}$ exists at all Péclet numbers with the solute concentration $c_{0}(r)=1 / r$. Whereas at high Péclet numbers, the coupled nonlinear advective term $\mathbf{u} \cdot \nabla c$ would lead to a polarization of the concentration around the particle surface, thus any infinitesimal perturbation to the immobile state will trigger a spontaneous autophoretic swimming motion. Although this symmetry-breaking behavior was reported in [2] where an axisymmetric constraint is imposed, we need to emphasize that, we have released such a constraint and attempt to explore all possible equilibriums of the entire system in the full three-dimensional simulations.

Since the considered problem is relevant to spherical geometry, it is ideal to use the Lamb's general solution in Stokes flow [1]. By applying the present setup, the velocity field can be analytically expressed through spherical harmonics expansion:

$$
\begin{aligned}
& u_{r}=\sum_{n=1}^{\infty} \sum_{m=0}^{n} \psi_{n}(r)(n+1) P_{n}^{m}(\cos \theta)\left(B_{m n} \cos m \phi+\tilde{B}_{m n} \sin m \phi\right), \\
& u_{\theta}=\sum_{n=1}^{\infty} \sum_{m=0}^{n} \chi_{n}(r) \sin \theta P_{n}^{m^{\prime}}(\cos \theta)\left(B_{m n} \cos m \phi+\tilde{B}_{m n} \sin m \phi\right), \\
& u_{\phi}=\sum_{n=1}^{\infty} \sum_{m=0}^{n} \chi_{n}(r)\left(\frac{-m P_{n}^{m}(\cos \theta)}{\sin \theta}\right)\left(\tilde{B}_{m n} \cos m \phi-B_{m n} \sin m \phi\right),
\end{aligned}
$$

where $\psi_{1}(r)=\frac{2}{3}\left(1-\frac{1}{r^{3}}\right), \psi_{n}(r)=\frac{1}{r^{n}}\left(1-\frac{1}{r^{2}}\right)$ for $n \geq 2, \chi_{1}(r)=\frac{2}{3}\left(-2-\frac{1}{r^{3}}\right)$, and $\chi_{n}(r)=\frac{1}{r^{n}}\left(\frac{n-2}{n}-\frac{1}{r^{2}}\right)$ for $n \geq 2$, and the prime in $P_{n}^{m}$ represents the differentiation. Representing the solute concentration on the surface in Fourier modes along the azimuthal direction by

$$
c(1, \theta, \phi, t)=\sum_{m=0}^{\infty}\left(D_{m}(\theta, t) \cos m \phi+\widetilde{D}_{m}(\theta, t) \sin m \phi\right)
$$

and using the prescribed slip boundary condition, we can obtain the coefficients in Eq. (11) through Eqs. (82, 90-93) in Ref. 11 as

$$
\begin{aligned}
& B_{m n}=\frac{1}{4} \frac{(2 n+1)(n-m) !}{(n+1)(n+m) !} \int_{-1}^{1} n(n+1) D_{m}(\mu, t) P_{n}^{m}(\mu) \mathrm{d} \mu \\
& \tilde{B}_{m n}=\frac{1}{4} \frac{(2 n+1)(n-m) !}{(n+1)(n+m) !} \int_{-1}^{1} n(n+1) \tilde{D}_{m}(\mu, t) P_{n}^{m}(\mu) \mathrm{d} \mu
\end{aligned}
$$

where we have used the notation $\mu=\cos \theta$. From the above equations $(10)-(13)$, we can see that the velocities $\mathbf{u}$ and $\mathbf{U}$ are given in terms of the concentration field. Substituting Eq. (11) into the concentration equation (9) yields a closed nonlinear equation for $c$. 
By plugging-in the analytic velocity solution (11) at $r=1$ to Eq. (10) gives the phoretic translational speed $\mathbf{U}=\frac{4}{3}\left(B_{11} \mathbf{e}_{x}+\tilde{B}_{11} \mathbf{e}_{y}-B_{01} \mathbf{e}_{z}\right)$. One can see that only the first harmonic modes $B_{11}, \tilde{B}_{11}$, and $B_{01}$ are responsible for the swimming velocity in the $x-, y$-, and $z$-directions, respectively, and this allows the phoretic particle to swim freely in the three-dimensional domain.

\subsection{Numerical algorithm}

As aforementioned, by using the Lamb's solution for the velocity field, we only need to solve the nonlinear transportation equation for the concentration field. Since the considered domain is unbounded $(r \in[1, \infty))$, we can map the domain into a finite one $(\bar{r} \in(0,1])$ by applying Kelvin's inversion with $\bar{r}=1 / r$. The new concentration $\bar{c}(\bar{r}, \theta, \phi)=\frac{1}{\bar{r}} c\left(\frac{1}{\bar{r}}, \theta, \phi\right)$ fulfills

$$
\frac{1}{\bar{r}^{5}}\left(\bar{r} \frac{\partial \bar{c}}{\partial t}+\mathbf{u}(\bar{c}) \cdot \bar{\nabla} \bar{c}\right)=\frac{1}{P e} \bar{\Delta} \bar{c}
$$

where $\bar{\nabla}=\left(-\bar{r}^{2}-\bar{r}^{3} \frac{\partial}{\partial \bar{r}}, \bar{r}^{2} \frac{\partial}{\partial \theta}, \frac{\bar{r}^{2}}{\sin \theta} \frac{\partial}{\partial \phi}\right)$ and the Laplace operator $\bar{\Delta}$ is preserved (see Eq. (8)). We employ a first-order implicit-explicit time advancing scheme to discretize Eq. (14), that is, we treat the nonlinear advective term explicitly while the diffusive component is handled implicitly:

$$
\frac{1}{\bar{r}^{5}}\left(\bar{r} \frac{\bar{c}^{n+1}-\bar{c}^{n}}{\Delta t}+\mathbf{u}\left(\bar{c}^{n}\right) \cdot \bar{\nabla} \bar{c}^{n}\right)=\frac{1}{P e} \bar{\Delta} \bar{c}^{n+1},
$$

where the superscript on a variable represents the time level index and $\Delta t$ is the time step size. The spatial discretization for all numerical variables is defined as stated in Section 2. For the computation of the velocity $\mathbf{u}\left(\bar{c}^{n}\right)$, firstly we need to calculate $D_{m}$ and $\tilde{D}_{m}$ in Eq. 12 by applying Fast Fourier Transform (in a truncated sense with the highest frequency mode $(p+1)$ ), and then use them to find $B_{m n}$ and $\tilde{B}_{m n}$ in Eq. (13) in which the integrations can be accurately approximated by Gaussian quadrature. Finally, those coefficients $B_{m n}$ and $\tilde{B}_{m n}$ are substituted into Eq. 11) to obtain the velocity (truncated at the highest harmonic mode of order $p$ ) with a change of variable from $r$ to $\bar{r}$. The spatial derivatives in the gradient term $\bar{\nabla} \bar{c}^{n}$ is approximated by central difference in the radial direction while a spectral method is used in the polar and azimuthal directions (see 6] for detail). Equation $\sqrt{15}$ forms a Helmholtz equation for $\bar{c}^{n+1}$ which can be efficiently solved by the method proposed in Section. 2 .

\subsection{Numerical simulations}

To investigate the spontaneous locomotion of the phoretic particle, we perform the simulations at various Péclet numbers where the concentrations being initially set as $c_{0}(r)=1 / r$ (immobile solution) with a small perturbation on the particle surface. For all the present simulations, we set 32 grid points in the radial direction, 32 and 64 points in the polar and azimuthal directions, respectively; the time step size $\Delta t$ is set to be of the same order as $\Delta \bar{r}$ to maintain the numerical stability.

Our numerical results reveal that, for small $P e$ numbers, the system recovers to the immobile solution regardless of the imposed perturbation, whereas when $P e$ is sufficiently large, the interplay between the advection and diffusion terms triggers a spontaneous symmetry-breaking swimming motion along a given direction (fixed by the initial perturbation) with a constant speed. As an example, the snapshots of the concentration distribution at different times for $P e=10$ are demonstrated in Fig. 1. After a long time, a comet-like pattern is formed (see $T=2000$ ) and the concentration field is axisymmetric about the direction of the phoretic velocity $\mathbf{U}$. In addition, it is found that the symmetry-breaking solution occurs in the form of a supercritical bifurcation when $P e>P e_{c}=4$; the particle sets into a directed motion with the long-time swimming velocity $\|\mathbf{U}\|$. We show the velocity as a function of the Péclet number in Fig. 2. The nonlinear variation of the phoretic velocity agrees quantitatively with the ones obtained under axisymmetric assumption in [2].

Interestingly, by increasing further the Péclet number reveals a new instability: the particle quits the unidirectional swimming motion and enters into an apparently chaotic regime. As an example, see Fig. 3 for the numerical results at $P e=24.5$. It can be seen that the particle exhibits an apparently random 

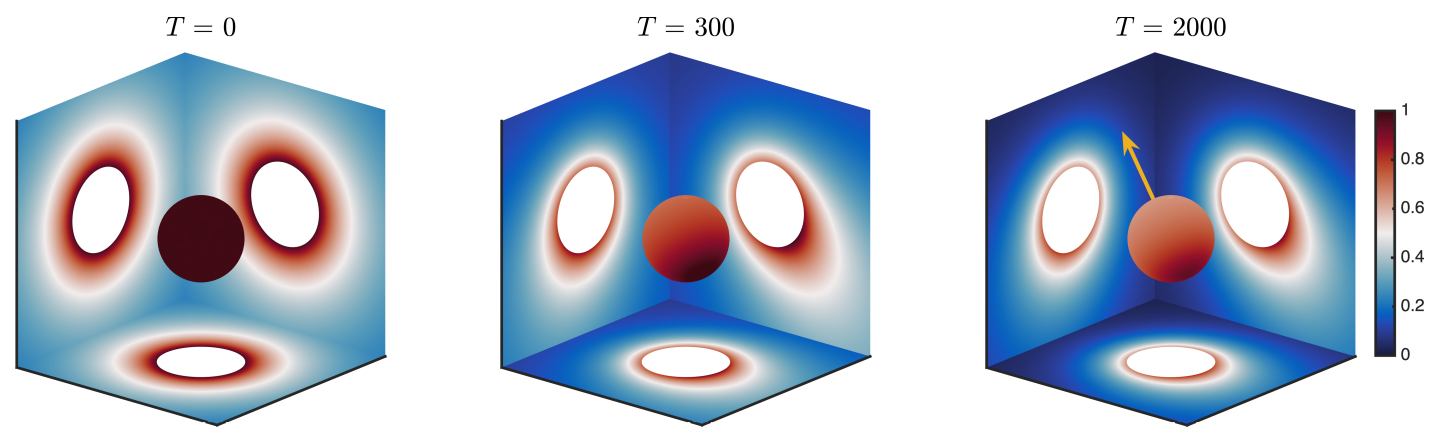

Figure 1: The snapshots of the concentration distribution on the surface of the particle and on the planes of symmetry at different times for $P e=10$. At $T=2000$, the particle attains a symmetry-breaking self-propelled motion along a straight direction (indicated by the yellow arrow) with the constant phoretic speed $\|\mathbf{U}\|=8.634 \times 10^{-2}$.

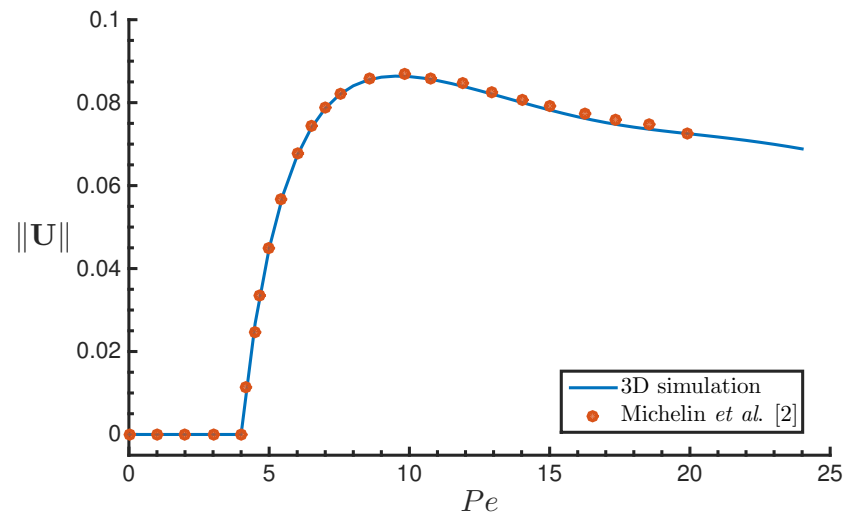

Figure 2: The bifurcation diagram for the phoretic velocity $\|\mathbf{U}\|$ as a function of $P e$. Our results (solid line) are in quantitative agreement with the ones obtained under axisymmetric assumption [2] (red dots).

motion (see the trajectory in Fig. 3(a)) and we quantify this random walk by measuring the mean square displacement $\operatorname{MSD}(\tau)=<\|\mathbf{r}(t+\tau)-\mathbf{r}(t)\|^{2}>$, where $\mathbf{r}(t)$ means the location of the particle at time $t$ and the notation $\langle\cdot\rangle$ computes the average along the entire trajectory. The MSD result is given in Fig. 3(b) as the solid blue line. For short times the particle attains a persistent run-and-tumble-like motion where the MSD is quadratic in time, while at longer times, due to the random turns of swimming directions a de-correlation process takes place, the MSD shows a linear behavior. This demonstration gives evidence that the complicated swimming motion can arise under the minimal version of dynamics such as in this system, a strong nonlinearity induced by the advective component. There are still interesting questions which require further investigation and we leave them as future works.

\section{Conclusions}

In this note, a fast direct solver for Poisson equation was developed in spherical geometry with finite and infinite domains. Representing a solution by spherical harmonics expansion, we only need to solve a set of one-dimensional mode differential equations. From the numerical point of view, this step reduces significantly the numerical complexity of the problem. We use finite difference method to discretize those mode differential equations with a proper choice of the radial grid layout that is shifted half mesh away from the origin. In this way, the pole singularity can be naturally avoided. We deal with Poisson equation in an infinite domain by using Kelvin's inversion which maps the domain into a finite one with the perfect preservation of the Laplace operator. As a result, fast solvers can be easily applied. A numerical verification 

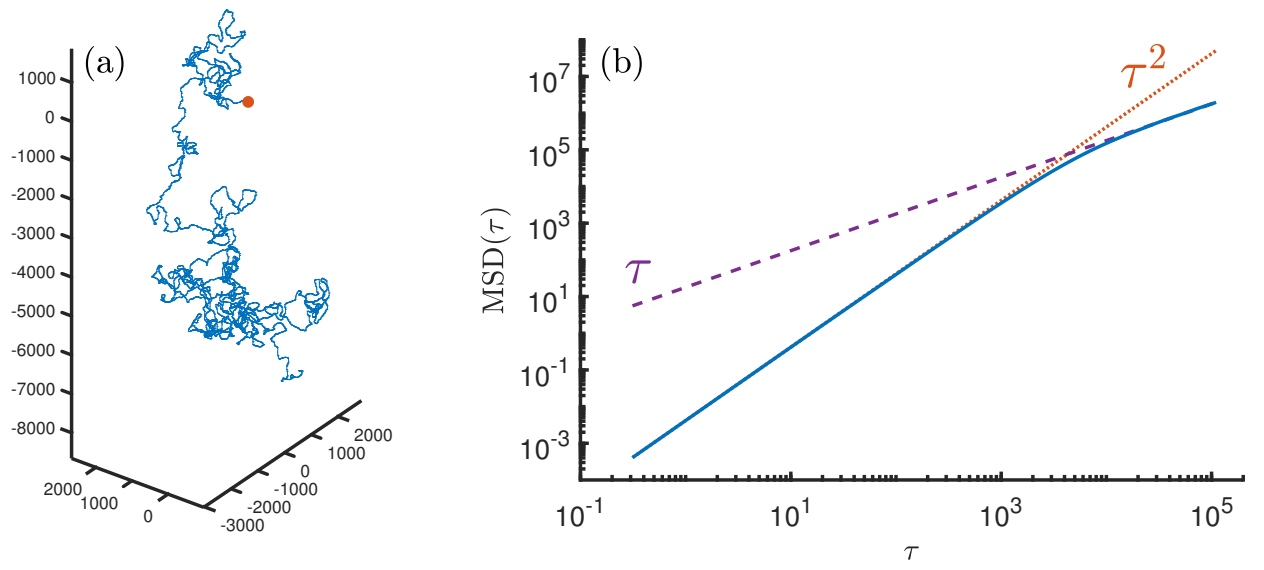

Figure 3: (a) The erratic particle trajectory at $P e=24.5$ (the red dot marks the origin). (b) The corresponding MSD (solid line) shows a classical random walk as normal diffusion.

is conducted to illustrate the accuracy and efficiency of the method. The proposed fast Poisson solver is then applied to study the diffusiophoretic system. The emergence of complex dynamics going from straight swimming to chaotic motion for a rigid isotropic particle in a purely phoretic fluid medium has been identified. The present results may serve as a guide for future systematic analyses for diffusiophoretic problems.

\section{Acknowledgement}

We would like to thank S. Rafai for the inspiring discussions. T.-S. Lin and W.-F. Hu acknowledge support by Ministry of Science and Technology, Taiwan, under research grant MOST-107-2115-M-009-008-MY2 and MOST-107-2115-M-005-004-MY2, respectively. C. Misbah thanks CNES (Centre National d'Etudes Spatiales) and the French-German university program "Living Fluids" (grant CFDA-Q1-14).

\section{References}

[1] W. Paxton et al., Catalytic nanomotors: autonomous movement of striped nanorods, J. Am. Chem. Soc. 126 (2004) 13424.

[2] S. Michelin, E. Lauga, D. Bartolo, Spontaneous autophoretic motion of isotropic particles, Phys. Fluids 25 (2013) 061701.

[3] J. Shen, Efficient spectral-galerkin methods iv. spherical geometries, SIAM J. Sci. Comput. 20 (4) (1999) 1438-1455.

[4] M.-C. Lai, W.-W. Lin, W. Wang, A fast spectral/difference method without pole conditions for poisson-type equations in cylindrical and spherical geometries, IMA J. Numer. Anal. 22 (2002) 537-548.

[5] M. Abramowitz, I. A. Stegun, Handbook of Mathematical Functions: with Formulas, Graphs, and Mathematical Tables, Dover Publications, 1965.

[6] J. C. Adams, P. N. Swarztrauber, Spherepack (2011).

URL https://www2.cisl.ucar.edu/resources/legacy/spherepack

[7] S. Orszag, Fourier series on spheres, Mon. Weather Rev. 102 (1974) 56-75.

[8] J. Wermer, Potential Theory, Springer, 1981.

[9] J. L. Anderson, Colloid transport by interfacial forces, Ann. Rev. Fluid Mech. 21 (1989) 61-99.

[10] H. A. Stone, A. Samuel, Propulsion of microorganisms by surface distortions, Phys. Rev. Lett. 77 (1996) $4102-4104$.

[11] O. S. Pak, E. Lauga, Generalized squirming motion of a sphere, J. Eng. Math. 88 (2014) 1-28. 